\title{
Osteogenic efficiency in vivo of scaffolding material of prefabricated vascularization
}

\author{
XIAORUI JIANG ${ }^{1 *}$, HUIYING YANG ${ }^{2 *}$, BOTAO HUANG ${ }^{1}$, GUODONG LIN ${ }^{1}$, \\ KAI WANG ${ }^{1}$, YONGCHUN MENG ${ }^{3}$ and CHUNYU XUE ${ }^{4}$ \\ ${ }^{1}$ Department of Orthopedics, The Affiliated Yantai Yuhuangding Hospital of Qingdao University; \\ ${ }^{2}$ Department of Hepatology, Yantai Infectious Disease Hospital; ${ }^{3}$ Department of Orthopedics, \\ The Affiliated Yantai Hospital of Binzhou Medical University, Yantai, Shandong 264000; \\ ${ }^{4}$ Emergency Department, Qingdao Municipal Hospital, Qingdao, Shandong 266011, P.R. China
}

Received December 6, 2017; Accepted October 24, 2018

DOI: $10.3892 /$ etm.2018.6915

\begin{abstract}
Osteogenic efficiency of pre-vascularization and non-vascularization decalcified bone scaffolds in bone defect repair was investigated. Twenty-one Sprague-Dawley (SD) mice were randomly assigned to three groups, and a bone defect area of $\sim 1.5 \mathrm{~cm}$ in length in the thigh bone of the right posterior limbs of each mouse was made. Pre-vascularization decalcified bone scaffolds in vitro (group A) and non-treatment decalcified bone scaffolds (group B) were separately implanted. The defect vacancy was considered as the blank control (group C). Sampling was made 4 weeks after the operation for the histological examination, and then the osteogenic efficiency was observed by gross sample, imaging, hematoxylin and eosin staining and Masson's staining. When implanting pre-vascularization decalcified bone scaffolds in vitro, the scaffolding material showed an obvious absorption, and more new bone formations and abundant vascular proliferation were observed. In non-vascularization decalcified bone scaffolds implanting, absorption insufficiency of the scaffolding material was observed, fewer new-born bone formations were shown, and the new vessels were very small and few in number. The pre-vascularization decalcified bone scaffolds had a better osteogenic efficiency.
\end{abstract}

\section{Introduction}

Extensive bone defect is frequently encountered in the treatment of orthopedic trauma, tumors, infection and some

Correspondence to: Dr Chunyu Xue, Emergency Department, Qingdao Municipal Hospital, 1 Jiaozhou Road, Qingdao, Shandong 266011, P.R. China

E-mail: kea886886@163.com

${ }^{*}$ Contributed equally

Key words: pre-vascularization decalcified bone scaffolds, decalcified bone scaffolds, bone defect, osteogenic efficiency congenital diseases and it is hard to deal with. The traditional methods for bone defect repair, such as autogenous, allogeneic and heterogeneous bone transplantations, are clinically limited since extra trauma can be caused, and there is limited supply and immunogenicity thereof. However, the bone tissue engineering technology with the model of 'scaffolding material plus seed cell plus growth factor' provides a new pathway for solving the above problems (1). Scaffolding material with both the osteoconduction and osteoinductivity has been used to load autologous seed cells for repairing the extensive bone defect. This solves the clinical issue, improves technological and industrialization development, leading to significant socioeconomic benefits.

The interaction between angiogenesis and osteogenesis is a complex process when considering the growth and development of bones and regeneration and repair of bone trauma. So far the key factor for the difficult repair of extensive bone defect is the deficiency in sources of blood supply of specific anatomic sites. Therefore, the tissue-engineered bone vascularization is the base to ensure the survival of the transplants, and constitutes the key technology for the bone tissue engineering (2-4). Given that the decalcified bone scaffolds reserve the natural network multi-pore structure of cancellous bone, they are well-characterized by degradability and biocompatibility (5). In order to further study the transplantation effect of decalcified bone scaffolds after pre-vascularization, the bone defect repair in the posterior limbs of mice implanted with pre-vascularization decalcified bone scaffolds in vitro and non-treatment decalcified bone scaffolds, and the osteogenic efficiency for the two scaffold materials were evaluated.

\section{Materials and methods}

Experimental materials. A total of 21 male Sprague-Dawley (SD) mice (8-12 weeks old, weighing 250-350 g) were obtained from Beijing Vital River Laboratory Animal Technology, Co., Ltd. (Beijing, China). All mice were housed in controlled conditions $\left(12 \mathrm{~h}\right.$ light/dark cycle, $22 \pm 0.5^{\circ} \mathrm{C}, 50-60 \%$ relative humidity) and they were fed with commercial rodent chow and tap water ad libitum. After 7 days of adaptive feeding, the mice were randomly divided into group A, B and C. Decalcified 
bone scaffolds with pre-vascularization in vitro and decalcified bone scaffolds were used.

Experimental methods. Thigh bone defect model of mice and material implantation were prepared. Twenty-one SD mice were randomly assigned to group A, B and C, and anesthesia was induced with intraperitoneal injection of $2 \%$ of pentobarbital sodium $(0.02 \mathrm{ml} / 100 \mathrm{~g})$. After local hair cropping in the right posterior limb of each mouse and disinfection with iodophor, the operating knife was used to cut the muscle layer open. Kirschner wire was adopted to make a bone defect area of $\sim 1.5 \mathrm{~cm}$ in length in the thigh bone. Decalcified bone scaffolds with pre-vascularization in vitro were implanted in the broken bones for group A; decalcified bone scaffolds were implanted in the broken bones for group B; and no material was implanted for group C. Tight suturing of all layers was carried out followed by disinfection. Intramuscular injection with 40,000 units of penicillin $G$ and sodium salt was given into the thigh after the operation and the mice were fed in separate cages. The study was approved by the Ethics Committee of the Affiliated Yantai Yuhuangding Hospital of Qingdao University (Yantai, China).

Gross observation. The postoperative daily life of mice was observed. At 4 weeks after operation the sacrifice of all groups of mice was conducted, and the sites with implanted materials were opened layer by layer. The peripheral tissues and the growth of the injured parts were observed.

Imaging observation. After the sacrifice of mice (4 weeks) the corresponding posterior limbs of all mice were taken down, and micro-CT (SkyScan 1176; Bruker microCT, Kontich, Belgium) was performed to scan the thigh bones of mice (scanning resolution: $18 \mu \mathrm{m}$, rotation angle: $360^{\circ}$, increase of rotation angle: $0.72^{\circ}$, voltage: $88 \mathrm{kV}$, power: $40 \mathrm{~W}$, electric current: $80 \mu \mathrm{A}$, average number of frames: 6 , binning: $1 \mathrm{x} 1$ ). Then, the repair of sites with bone defect was observed in all groups to determine whether they had synostosis and bone tissue molding.

Histopathological examination. The thigh bones of mice were taken after the sacrifice of mice, at 4 weeks after operation, and peripheral muscle tissues of bone tissues were removed. Neutral formalin (10\%) was used for fixation for 3 days, after which $10 \%$ of ethylenediamine tetraacetic acid (EDTA, pH 7.2) was used for decalcification for 1 month. Then, the sections were paraffin-embedded and H\&E and Masson's staining was performed. New bone formation, inflammatory response, bone cell growth, angiogenesis, material degradation, calcium deposition and ossein growth were observed.

\section{Results}

Gross observation and analysis results. The mice woke 30 min after the operation. On the day of operation all sites moved freely except for the operation site, and normal feeding was observed. After several days of postoperative observation, no infection was shown in the sutured sites, good healing was observed and there were no deseased mice, indicating a successful operation of material implantation and good biocompatibility for the materials without inducing any immunological rejection. After the sacrifice of the mice, the operation site was opened layer by layer. No swelling and water percolation were observed, and normal growth of the muscular tissues was shown, further suggesting good biocompatibility of the materials. In group A, the defect areas of the thigh bones of all mice were healed with no obvious difference from the other bone tissues, and the implanted scaffolds had completely degraded. In group B, the defect areas of the thigh bones of all mice were basically healed, and the regenerated bone tissues in other defect areas had no obvious differences from the other bone tissues, except for that part of cortical bones that were completely repaired, and the implanted scaffolds had completely degraded. In group $\mathrm{C}$, the defect areas of the thigh bones of all mice were filled with fibrous tissues, and no similar structure of bone tissues was observed in the defect areas. The results showed that osteanagenesis occurred in the defect areas of the thigh bones of the mice in group A, and bone defect areas were completely replaced by new bone tissues, suggesting a complete repair for defect areas. Steanagenesis also occurred in the defect areas of the thigh bones of mice in group B, and most of bone defect areas were replaced by new bone tissues, suggesting a basic repair for defect areas. In group $\mathrm{C}$, no osteanagenesis occurred in the defect areas of thigh bones of mice, and bone defect areas were replaced by muscular tissues or fibrous tissues.

Imaging observation and analysis results. Fig. 1 shows the three-dimensional reconstruction of defect areas scanned by micro-CT. As can be seen from Fig. 1A, the defect area was not obvious in group A and it was completely replaced by new bone tissue. Full connection of cortical bone was shown and no development for implanted materials was observed, indicating a complete degradation of the materials. Fig. 1B shows that the edge of bone defect area was not obvious in group B and most areas were replaced by new bone tissues. The cortical bone showed no full connection, and no development was observed for implanted materials, indicating a complete degradation of materials. Fig. 1C shows that a small number of new bone tissues were formed in the edge of bone defect area in group C, the defect area was obvious, and it was not filled or replaced by new bone tissues. The results revealed that the decalcified bone scaffolds with pre-vascularization in vitro implanted in the bone defect areas of mice can quickly induce the blood into the inner scaffold and provide abundant nutrient substances and mesenchymal stem cells for the defect areas, benefiting the differentiation of mesenchymal stem cells into osteoblasts and osteanagenesis in defect areas. Owing to the reservation of three dimensional structure and collagen scaffold of original bone tissues, the treated decalcified bone scaffolds can provide better scaffold for cell crawling with the same and better effect of inducing osteogenesis. However, it was lower in the effect of inducing osteogenesis as compared with decalcified bone scaffolds with pre-vascularization in vitro.

Histopathological examination and analysis results. To evaluate the repair of sites with bone defect, sampling was conducted using H\&E staining detection, the results of which can be seen in Fig. 2. At 4 weeks after operation, the mice in groups A and B showed an obvious recovery sign, 
A

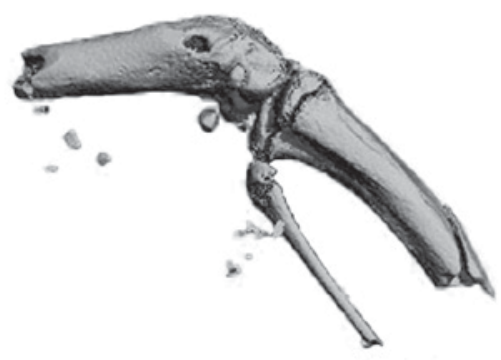

B

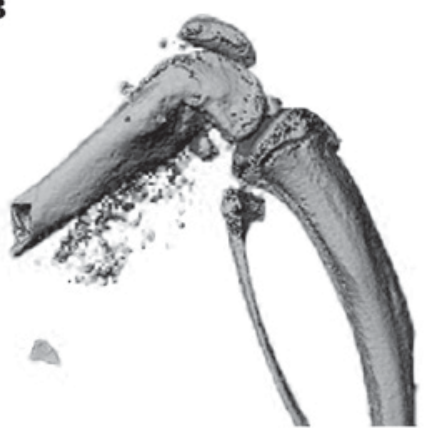

C

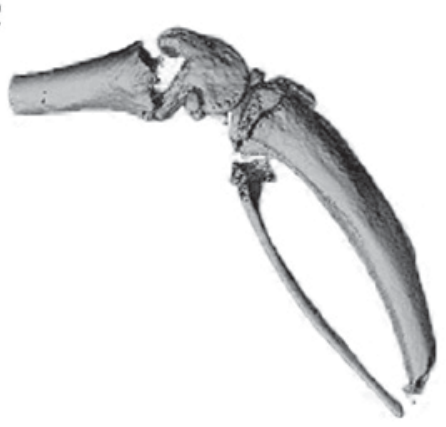

Figure 1. Three-dimensional reconstruction of bone defect repair of posterior limbs of mice. (A) Decalcified bone scaffolds with pre-vascularization in vitro. (B) Decalcified bone scaffolds. (C) Blank.
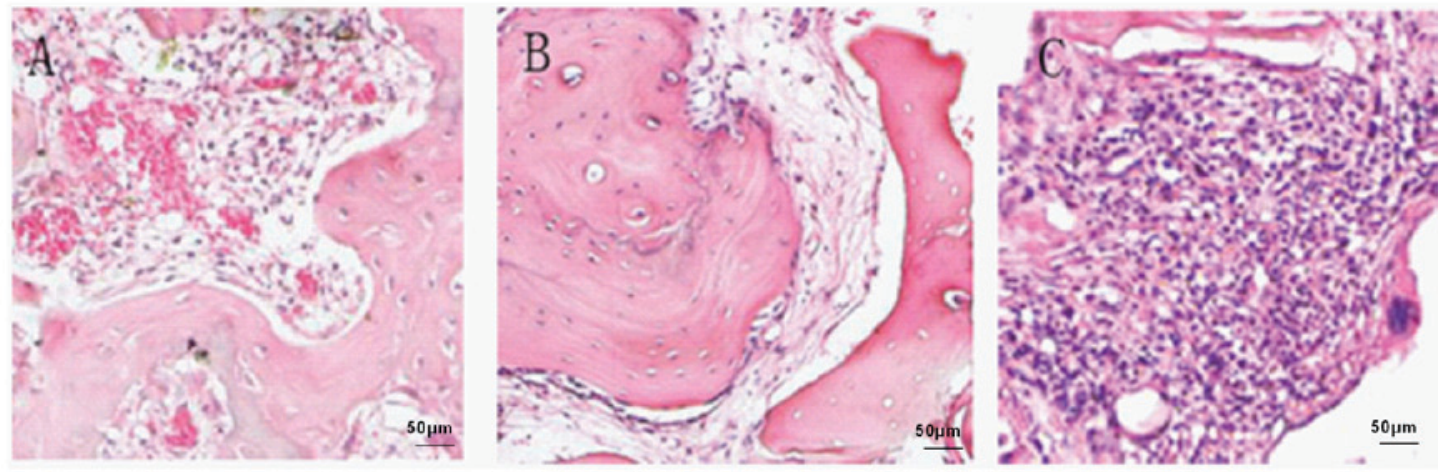

Figure 2. H\&E staining results of bone defect repair of posterior limbs of mice. (A) Decalcified bone scaffolds with pre-vascularization in vitro. (B) Decalcified bone scaffolds. (C) Blank.
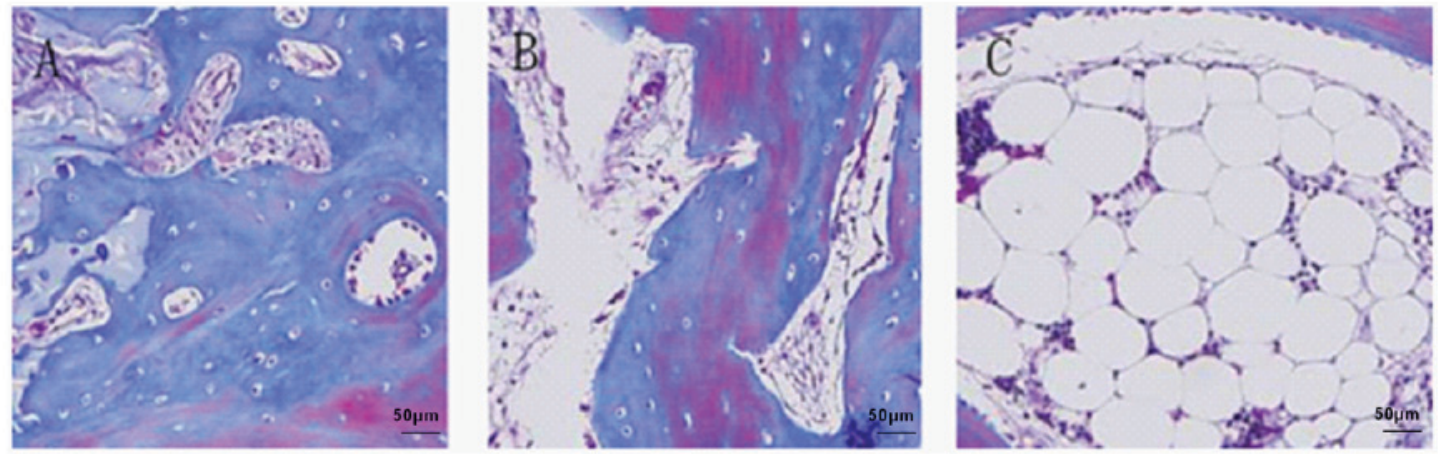

Figure 3. Masson's staining results of bone defect repair of posterior limbs of mice. (A) Decalcified bone scaffolds with pre-vascularization in vitro. (B) Decalcified bone scaffolds. (C) Blank.

osteoblasts were shown in the peripheral area, and a small number of structures of bone trabeculas and marrow cavity with no complete remodeling were observed (Fig. 2A and B). The scaffold materials in group A (Fig. 2A) were completely degraded and absorbed, the aggregation of vast osteoblasts and undifferentiated mesenchymal cells was seen in the surface of new bones, and connective tissues accompanied by abundant angiopoiesis were observed in the periphery. A relatively small number of new-born bones grew into the implanted bones in a finger-like form with basically no neovascularization in group B (Fig. 2B). The blank control group with no implantation of material (Fig. 2C) showed no sign of repair. Plenty of fat vacuoles, fibrous tissues and other tissues had filled the defect areas, preventing the process of bone repair. The implantation of pre-vascularization decalcified bone scaffolds and decalcified bone scaffolds not only promoted the adhesion, recruitment and growth of osteoblasts in the initial stage of repair, but also prevented the invasion of cells and tissues (such as epithelial cells, fibroblasts or connective tissues) irrelevant to osteogenesis to some extent, providing a favorable growing environment for the bone repair. No inflammatory cell infiltration was observed in any of the groups.

After the samples receiving Masson's compound staining, the collagenous fiber was showed baby blue, new bone blue, cell nucleus black blue, erythrocyte salmon pink and decalcified bone red (Fig. 3). In the 4th week after implantation, obvious 
bone repair signs and accumulation of collagenous fiber tissues were shown in the defect sites of the mice in groups A and $\mathrm{B}$ and vast new bone tissues had filled the defect sites and had better integration with peripheral normal old bone tissue. In group A (Fig. 3A) the repair effect was relatively better than group B (Fig. 3B), an obvious absorption of scaffold materials was shown, more new bones and abundant vascular proliferation. However, for the blank control group (Fig. 3C) with no implantation of any material, some fibrous connective tissues had accumulated in the defect sites and the sign of bone repair was not obvious. This experiment further showed that decalcified bone scaffolds with pre-vascularization treatment implanted in the bone defect sites have better repair effect and osteoconductive regeneration capacity.

\section{Discussion}

With the continuous development of bone tissue engineering technology, the extensive bone defect repair by use of tissueengineered bone has been a current research hotspot (6-8). Considerable number of theories and techniques have been accumulated in the field of bone tissue engineering. However, the speed and quality of structuring tissue-engineered bones using the existing technologies do not meet the clinical requirements, limiting further development and final clinical application of bone tissue engineering. The important reason is that the existing technologies cannot ensure the early survival in vivo of seed cells loaded by scaffold materials, thereby exerting an influence on its long-term osteogenic efficiency in vivo.

An effective way for solving the above problems is abundant vasoganglions formed quickly within scaffold materials $(8,9)$. The seed cells need to take in oxygen and nutrient substances through blood vessel and transfer metabolites. For extensive bone defects, larger volumes of implanted tissueengineered bone are needed, so the vascularization in the scaffold materials can play a vital role. A study has shown that osteoblasts can only survive in the region, $100 \mu \mathrm{m}$ away from blood vessel (10). Therefore, the way to build the vascularized tissue-engineered bone in a fast and effective way has become a hot issue.

Researchers worldwide have put forward some methods of vascularization construction from the perspectives of three elements of tissue engineering, such as seed cells used for the construction of blood vessel and bone jointly implanted in scaffold materials, the adoption of growth factors for stimulating or relevant genes for transfecting seed cells, and the preparation of biological materials suitable for the angiogenesis. Although, some effects have been achieved, an effective way for building tissue-engineered bone with full vascularization is lacking, and the reason for this is that peripheral capillaries had not grown in the early stage when tissue-engineered bone was implanted in vivo. This stage is important for the seed cell survival and proliferation, and there is a certain time difference between the vascularization and osteogenesis. Some studies used the property of the vascular endothelial cells to form immature tubular structures in the scaffold materials and implanted the endothelial cells in the scaffold materials in advance, and after certain vasoganglions were formed in vitro, they were implanted in vivo to build tissue-engineered skins (11). It was observed that the tubular structure can fuse and crosslink with autologous vessels of the host. Some scholars applied this to the construction of tissue engineered bone and the method of 'pre-implantation' of vascular endothelial cells was adopted for 'pre-vascularization' of scaffold materials (12). This novel method has provided a new idea for solving the problem of early survival in vivo of osteogenic seed cells, which, meanwhile, has conformed to the purpose of the construction of vascularized tissue-engineered bone with complete tissue structure.

The osteogenic efficiency of decalcified bone scaffolds with pre-vascularization in vitro and decalcified bone scaffolds was investigated in this study. The results showed that decalcified bone scaffolds with pre-vascularization in vitro are obviously better than the experimental group of decalcified bone scaffolds in osteogenic efficiency. The reason for this is that owing to the reservation of three dimensional structures and collagen scaffold of original bone tissue, decalcified bone scaffolds can provide a better scaffold for the cell crawling, and after decalcified bone scaffolds with pre-vascularization in vitro are implanted in the bone defect areas of mice, they can induce the blood into the scaffold, supplying the oxygen and nourishments needed by tissue repair, promoting the generation and calcification of new bone tissues, gradually replacing implanting materials, accelerating the degradation of implanting materials, and finally completing the repair process to make the defect areas filled with bone tissues and achieve the complete connection with peripheral tissues. Therefore, the vascularization of bone tissue engineering is an effective way to deal with the problem of low oxygen environment in the process of bone defect repair, and as the seed cells, growth factors and scaffold materials, it has become the determining factor for the successful repair of bone tissue engineering.

\section{Acknowledgements}

Not applicable.

\section{Funding}

The present study was supported by the Shandong Natural Science Foundation (ZR201808170124), Yantai Science and Technology Develpment (2019YT06000140), and the National Natural Science Foundation of China (81301570).

\section{Availability of data and materials}

The datasets used and/or analyzed during the present study are available from the corresponding author on reasonable request.

\section{Authors' contributions}

$\mathrm{XJ}$ and HY were involved in the conception of the study. BH and GL were also involved in the conception and design of the study. KW contributed to the analysis and interpretation of the data. YM assisted with the histopathological examination. CX helped to perform the analysis, and offered constructive discussions. All authors read and approved the final manuscript. 


\section{Ethics approval and consent to participate}

The study was approved by the Ethics Committee of the Affiliated Yantai Yuhuangding Hospital of Qingdao University (Yantai, China).

\section{Patient consent for publication}

Not applicable.

\section{Competing interests}

The authors declare that they have no competing interests.

\section{References}

1. Yousefi AM, James PF, Akbarzadeh R, Subramanian A, Flavin C and Oudadesse H: Prospect of stem cells in bone tissue engineering: A review. Stem Cells Int 2016: 6180487, 2016.

2. Zhao T and Sun H: Insight into bone tissue engineering scaffold materials and their vascularization. Zhongguo Zu Zhi Gong Cheng Yan Jiu Yu Lin Chuang Kang Fu 17: 6832-6838, 2013.

3. Nakano K, Murata K, Omokawa S, Akahane M, Shimizu T, Kawamura K, Kawate K and Tanaka Y: Promotion of osteogenesis and angiogenesis in vascularized tissue-engineered bone using osteogenic matrix cell sheets. Plast Reconstr Surg 137 $1476-1484,2016$.
4. Mercado-Pagán ÁE, Stahl AM, Shanjani Y and Yang Y: Vascularization in bone tissue engineering constructs. Ann Biomed Eng 43: 718-729, 2015.

5. Xu Z, Wang G and Wang F: Histocompatibility of demineralized bone matrix decorated with poly-L-lysine for scaffolds Orthopedic J China 16: 1493-1497, 2015.

6. Amini AR, Laurencin CT and Nukavarapu SP: Bone tissue engineering: Recent advances and challenges. Crit Rev Biomed Eng 40: 363-408, 2012.

7. Zhou J, Lin H, Fang T, Li X, Dai W, Uemura T and Dong J: The repair of large segmental bone defects in the rabbit with vascularized tissue engineered bone. Biomaterials 31: 1171-1179, 2010.

8. Nguyen LH, Annabi N, Nikkhah M, Bae H, Binan L, Park S, Kang Y, Yang Y and Khademhosseini A: Vascularized bone tissue engineering: Approaches for potential improvement. Tissue Eng Part B Rev 18: 363-382, 2012.

9. Almubarak S, Nethercott H, Freeberg M, Beaudon C, Jha A, Jackson W, Marcucio R, Miclau T, Healy K and Bahney C: Tissue engineering strategies for promoting vascularized bone regeneration. Bone 83: 197-209, 2016.

10. Lovett M, Lee K, Edwards A and Kaplan DL: Vascularization strategies for tissue engineering. Tissue Eng Part B Rev 15: 353-370, 2009.

11. Bussolino F, Mantovani A and Persico G: Molecular mechanisms of blood vessel formation. Trends Biochem Sci 22: 251-256, 1997.

12. Thottappillil N and Nair PD: Scaffolds in vascular regeneration: Current status. Vasc Health Risk Manag 11: 79-91, 2015. Attribution-NonCommercial-NoDerivatives 4.0 International (CC BY-NC-ND 4.0) License. 